\title{
PROPUESTAS DE GRAFEMARIOS PARA LA LENGUA MAPUCHE: DESDE LOS FONEMAS A LAS REPRESENTACIONES POLÍTICO-IDENTITARIAS ${ }^{1}$ \\ Grapheme alphabet proposals for mapuche language: from phonemes to political and identity representations
}

\author{
Pilar Álvarez-Santullano Busch* \\ Amilcar Forno Sparosvich** \\ Eduardo Risco del Valle ${ }^{* * *}$
}

Resumen

En este artículo damos cuenta de las propuestas de grafemarios — más conocidas y diferenciadas entre sí- para escribir la lengua mapuche y discutimos sus fundamentos y las tensiones que subyacen en ellas. Con ello esperamos contribuir a abrir la actual discusión para una toma de conciencia de las alternativas posibles, de las representaciones que se encuentran en disputa y de lo que generan estas concreciones cuando se llevan al plano de la educación intercultural. La aparición de grafemarios mapuche huilliches y la defensa de unos y resistencia a otros muestra que incluso en aquellas zonas aparentemente más "objetivas y técnicas" de la lengua lo político tiene lugar, evidenciando que lo lingüístico está irrenunciablemente atado a las relaciones de poder que involucran a los sujetos y a las comunidades de habla.

Palabras clave: Grafemarios, Lengua mapuche, Fonología, Identidad territorial.

\begin{abstract}
In this paper, we discuss some of the different and best known grapheme alphabet proposals for writing the Mapuche language, and discuss their rationale and the tensions that underlie them. With this, we hope to contribute to open the current debate to a new awareness of possible alternatives about the representations that are in dispute and about what these concretions generate when they are used in intercultural education. The appearance of Mapuche Huilliche grapheme alphabets, the adoption of some of them and the resistance to others show that even in language areas seemingly more "objective and technical", politics takes place. This shows that linguistics is undeniably tied to power relations involving individuals and speech communities.
\end{abstract}

Key words: Grapheme alphabets, Mapuche language, Phonology, Territorial identity.

\footnotetext{
${ }^{1}$ Agradecemos a CONICYT por financiar el Proyecto Fondecyt 1120792 "Estudio fonológico de las variedades del mapuche o mapuzugun habladas en territorio huilliche", de cuyos resultados se nutre este artículo; al Núcleo Milenio "Centro de Investigación en Educación en Contexto Indígena" (UCT), y al Núcleo de Estudios Críticos: Discurso, Poder y Sociedad (Universidad de Los Lagos) por los espacios y aportes para formular y discutir este texto.
} 


\section{LOS GRAFEMARIOS}

La preeminencia de la oralidad sobre la escritura ha sido un aspecto especialmente subrayado en la lingüística de fines del siglo $\mathrm{XX}$, de hecho, Werner Abraham (Abraham, 176) — citando a Lyons - se refería al tema en 1981 en los siguientes términos: "la escritura no es más que una representación gráfica, secundaria, del lenguaje. La lengua escrita se deriva del traslado del habla a un medio visual, secundario", en donde es posible notar, ya desde la puntuación y en especial enfatizado por la doble aparición del adjetivo 'secundario', un claro acento en la oralidad como también — desafortunadamente - una en extremo simplificada alusión al dramático cambio que supone ese 'traslado del habla a un medio visual' que, visto desde la perspectiva de una lengua de tradición oral que se asoma a ese mismo 'traslado' y, más aún, desde la perspectiva de la sociedad que la usa, adquiere ribetes de tal magnitud que trastocan (y colisionan con, a veces) los aspectos más esenciales de la identidad histórica, cultural y política de esa misma sociedad, como ha ocurrido con la aparición de grafemarios para la lengua mapuche en Chile y la reacción de reivindicación autonomista que estos grafemarios han suscitado en el pueblo huilliche ${ }^{2}$.

El esfuerzo por asignar una representación gráfica a los rasgos fónicos segmentales de la lengua, sea con el noble objetivo que sea, hunde sus raíces en los tiempos más antiguos del devenir cultural de nuestra tradición global y manifiesta un continuum perfectivo que, con ajustes paulatinos, ha ido depurando y haciendo más eficiente el sistema por medio de una mayor precisión de las correspondencias entre elementos del ámbito de lo gráfico y elementos del ámbito fónico. Desde los pictogramas e ideogramas que encontramos con abundancia en la escritura china o mesoamericana, pasando por los primeros prototipos de fonogramas (unilíteros, bilíteros y trilíteros) que, combinados con ideogramas de existencia previa, dieron forma al sistema jeroglífico egipcio, hasta llegar por último al alfabeto fenicio, en el que ya se puede encontrar una clara predominancia de correspondencias (quasi)unívocas entre sonido y signo, asistimos a un extenso desfile de problemas y propuestas de solución diversas que han dejado, no obstante, su huella en todas las reformulaciones ulteriores. En efecto, y si quisiéramos trazar una línea sucesoria ideal de eventos, al pictograma ${ }^{3}$ ( $\mathrm{y}$ tras un cierto proceso de estilización paulatina que bien puede apreciarse en la textilería mapuche) sucede en algunos sistemas el ideograma, un símbolo o combinación de símbolos que representa ya una idea completa y que resulta funcional para la comunicación gráfica de eventos $\mathrm{y}$, por supuesto, para la

\footnotetext{
${ }^{2}$ El grupo huilliche constituye uno de los subgrupos del pueblo mapuche junto a los picunches, pehuenches, y moluches o aracucanos.

${ }^{3}$ Entendemos por "pictograma" una representación que, habiendo tenido alguna vez una base más "realista" (estéticamente hablando), se ha ido estilizando paulatinamente y, en muchos casos, compulsada por los límites que imponía el instrumento con el que se trazaban los dibujos, pero que conserva aún su carácter iconográfico.
} 
creación literaria. En un momento posterior y catalizado por las necesidades de plasticidad con las que la expresión escrita desafía al sistema, muchos ideogramas pierden de manera paulatina su relación unívoca con ideas específicas y adquieren una nueva relación con el o los sonidos que lo inauguran en la ejecución oral, dando origen a símbolos con valor silábico y también con valor consonántico, como puede apreciarse en el sistema jeroglífico (en especial en el período demótico), en el sistema cuneiforme, en el alfabeto griego e incluso en el alfabeto latino que hoy utilizamos ${ }^{4}$. El posterior salto cualitativo de este proceso es, evidentemente, el surgimiento del alfabeto fonético que, manifestando sus beneficios en el uso académico especializado, llega a transformarse en muchos casos en la norma hacia la que miran quienes diseñan o evalúan sistemas de escritura postulados para dar forma gráfica a las lenguas que aún no la tienen.

Por supuesto, como ya lo hemos señalado, este somero itinerario representa una secuencia abstracta y no es el que recorren todas las lenguas que tienden a adquirir forma escrita y no es el recorrido por la lengua mapuche en su proceso de (incipiente) alfabetización. En efecto, puesta en este contexto, la lengua mapuche pasa de manera directa desde una etapa de pictogramas a la circunstancia histórica de adoptar un alfabeto, es decir, un sistema de representación gráfica de la lengua que se apoye en una correspondencia unívoca entre símbolo y sonido, en el estado ${ }^{5}$ en que los sistemas disponibles se encuentran hoy para las lenguas en uso.

Los grafemarios tienen como antecedentes una tradición de alfabetización de las lenguas indígenas que en América reconoce dos fuentes básicas: la primera de ellas, la evangelización que suponía la necesidad de contar con un soporte escrito para trasladar el evangelio a las lenguas vernáculas. Los destinatarios de estos textos eran sobre todo los propios misioneros, por lo que los símbolos estaban al servicio de que estos entendieran la escritura para aprender la lengua. La segunda tradición viene de la escritura cotidiana de nombres y lugares (adosadas respectivamente a la competencia lingüística del funcionario estatal a cargo de ejercer el registro correspondiente). La primera de estas tradiciones cuenta con el apoyo de trabajos lingüísticos previos en particular desarrollados para este fin, en cambio la segunda reposa más bien sobre la intuición lingüística del escritor hispanohablante que debe enfrentar el hecho de transcribir una voz de otra lengua en la suya. Desde la primera vertiente, por ejemplo, la isla ubicada en Lago Ranco, provincia de Valdivia, se escribiría preferentemente como Wapi, mientras que en la segunda opción se

\footnotetext{
${ }^{4}$ Una clara manifestación de esto se encuentra en las consonantes geminadas del alfabeto español (como "ll") y en el grafema " $x$ ", que en realidad representa dos sonidos: [ks].

${ }^{5}$ Las lenguas en uso, como se sabe, poseen o adoptan sistemas alfabéticos en los que las correspondencias símbolo-sonido no son perfectamente unívocas, lo que genera un gran número de dudas y controversias entre los hablantes en la fase de instalación, controversias que se relacionan con aspectos fundamentales de la cultura toda vez que la instalación de un sistema de escritura supone una brusca modificación de las pautas pragmalingüísticas de la interacción verbal.
} 
escribiría Huapi $^{6}$ y, a diferencia del primer caso, se trata de escribir en castellano las voces que provienen del mapuche.

\section{LA ESCRITURA EN EL CONTEXTO MAPUCHE}

En las últimas décadas han surgido diversas iniciativas para proveer de escritura a la lengua mapuche. Este proceso, que moviliza a una sociedad oral hacia la situación de sociedad con escritura (en lo que a la lengua vernácula se refiere), remueve condiciones sociales previas a su emergencia, entre estas, esquemas y representaciones sociales del "sí mismo" como pueblo y de sus relaciones con el grupo social dominante históricamente provisto de escritura.

En este sentido, la tarea de escribir una lengua que hasta ese momento ha funcionado en y para la tradición oral, no solo apunta a vitalizar la lengua misma, sino también a posicionar a sus hablantes (y ahora escritores) en una nueva vinculación o posibilidad de contrato entre pueblos que disputan en un mismo espacio por mantener y desarrollar sus prácticas y políticas culturales e institucionales.

La presentación y discusión de propuestas de un sistema gráfico, que resulte funcional a la tarea asumida, es uno de los primeros pasos para el desarrollo escriturario en la lengua propia de un pueblo. En este contexto, han surgido diversas propuestas de grafemario para la escritura de la lengua mapuche, las que se inscriben en un proceso no exento de tensiones en y para las comunidades que forman parte de dicho pueblo, tensiones que relevan la escritura como un dispositivo técnico, pero también político.

En este artículo damos cuenta y confrontamos cuatro propuestas, tal vez las más difundidas, de grafemarios para escribir la lengua mapuche con dos propuestas surgidas desde la territorialidad huilliche, discutimos además sus fundamentos y las tensiones que entre ellas se originan. Esperamos con ello contribuir a la discusión acerca de las representaciones y posicionamientos que se encuentran en disputa, especialmente en el plano de la educación intercultural donde se actualizan estas concreciones.

Con este fin, hemos incluido, en un primer grupo, los grafemarios de Raguileo, Alfabeto Unificado, Azümchefe, Académicos Universidad Católica de Temuco y, en el segundo grupo, los grafemarios del cacicado huilliche y el de Salvador Rumian. El conjunto total conforma una muestra que atiende a la distribución geográfica y a la diversidad de origen de los grafemarios (p. ej., académico vs. comunitario). Los grafemarios de Raguileo, el Alfabeto Unificado y el Azümchefe son los más utilizados, aunque en diversos contextos; el grafemario de la UCT tiene una importante repercusión, ya que esta Universidad dicta la carrera de Pedagogía Básica Intercultural en Contexto Mapuche, de manera que es aprendido y utilizado por los

\footnotetext{
${ }^{6}$ Ambas posibilidades de escritura (Wapi y Huapi), en lo que parece ser una confusión, las utiliza Augusta (1916: XIV y XV) refiriéndose a la isla señalada.
} 
estudiantes; finalmente, la inclusión de grafemarios huilliches constituye un punto de vista interesante, pues sus proponentes enfrentan el problema de la representación de su propia variante dialectal.

\subsection{Grafemarios}

\subsubsection{Alfabeto Raguileo}

El grafemario Raguileo fue creado en 1982 por Anselmo Raguileo, intelectual que, según datos proporcionados por Ruby Raguileo (Kintupil, 2007), participó en los movimientos mapuches remarcando la importancia política de la mantención del idioma para la existencia del pueblo mapuche, se desempeñó como Profesor de Lengua Mapuche en el Instituto Pedagógico de la Universidad de Chile en la década del 50 y en 1973 se tituló de Ingeniero en Ejecución en Química. En la década del 80, de vuelta en Temuco, realizó trabajo de campo en comunidades mapuches de distintas regiones para un proyecto de lingüística del CAPIDE, y para probar la confiabilidad del alfabeto entre 1982 y 1990 realizó diferentes cursos de lengua mapuche en CAPIDE, Ad Mapu y la Sociedad Mapuche Newen. Concordamos con Wittig (Wittig, 2006) en que "el Alfabeto Raguileo se fundamenta en una posición de diferenciación y autonomía de la lengua mapuche en relación con el castellano, la lengua de la sociedad dominante", en lo que difiere fundamentalmente del Alfabeto Unificado.

El Alfabeto Raguileo (CONADI, 107) incluye seis vocales y 20 consonantes (Tabla 1).

Tabla 1. Alfabeto Raguileo

\begin{tabular}{|c|c|c|c|c|}
\hline $\mathrm{N}^{\mathrm{o}}$ & Letra & Fonema & Pronunciación & Ejemplos $^{7}$ \\
\hline 1 & $\mathrm{~A}$ & $/ \mathrm{a} /$ & Como vocal $a$ española. & akun llegué \\
\hline 2 & $\mathrm{E}$ & /e/ & Como vocal $e$ española. & niefun tenía \\
\hline 3 & I & /i/ & Como vocal $i$ española. & xogli flaco \\
\hline 4 & $\mathrm{O}$ & $/ \mathrm{o} /$ & Como vocal $o$ española. & ponwi adentro \\
\hline 5 & $\mathrm{U}$ & $/ \mathrm{u} /$ & Como vocal $u$ española. & kayu seis \\
\hline 6 & $\mathrm{~V}$ & $\mid \mathbf{i} / /^{8}$ & $\begin{array}{l}\text { Como } u \text { sin redondear los labios, } \\
\text { (variante [ə]). }\end{array}$ & antv sol \\
\hline 7 & $\mathrm{C}$ & $/ \mathrm{t} \mathrm{f} /$ & $\begin{array}{l}\text { Semejante al castellano } c h \text {. En el área } \\
\text { huilliche (sur) tiene también sonido } t s \text {. }\end{array}$ & ciwku tiuque \\
\hline 8 & Z & $/ \theta /$ & $\begin{array}{l}\text { Al centro del territorio mapuche, como } z \\
\text { española. Al norte y sur, como } d \text { suave [ð]. }\end{array}$ & casi sal \\
\hline 9 & $\mathrm{~F}$ & $/ \mathrm{f} /$ & $\begin{array}{l}\text { Como } f \text { castellana (centro). } \\
\text { Más cercana a } v \text { (norte). } \\
\text { Como } f \text {, como } j \text { o aspirada (sur). }\end{array}$ & yerfa yerba \\
\hline 10 & Q & $/ \gamma /$ & Suena como $g$ suave. & naqeltu abajo \\
\hline 11 & $\mathrm{~K}$ & $/ \mathrm{k} /$ & Como $c$ española antes de vocales $a \mathrm{o} u$. & akun llegué \\
\hline
\end{tabular}

\footnotetext{
${ }^{7}$ En todos los grafemarios hemos tratado de mantener ejemplos propuestos por los propios autores. Solo cuando no ha sido posible, hemos incorporado ejemplos provistos por otras fuentes.

${ }^{8}$ Este fonema suele ser representado mediante $/ \mathrm{i} /, / \mathrm{w} / \mathrm{o} / \mathrm{\partial} /$, lo que en general parece haber dependido de la variedad dialectal descrita.
} 


\begin{tabular}{|c|c|c|c|c|}
\hline 12 & $\mathrm{~L}$ & $/ 1 /$ & Como $l$ española. & alka gallo \\
\hline 13 & B & 1/ & $\begin{array}{l}\text { Interdental } \\
\text { En territorio huilliche, suele ser retrofleja }\end{array}$ & koyba mentiroso \\
\hline 14 & $\mathrm{~J}$ & $\mid \mathrm{K} /$ & $\begin{array}{l}\text { Como } l l \text { española más tradicional o como } \\
\text { habitualmente la } l l \text { de Chile }\end{array}$ & kawej caballo \\
\hline 15 & $\mathrm{M}$ & $/ \mathrm{m} /$ & Como $m$ española & kimvn saber \\
\hline 16 & $\mathrm{~N}$ & $/ \mathrm{n} /$ & Como $n$ española & koybatun mentir \\
\hline 17 & $\mathrm{H}$ & $/ \mathrm{n} /$ & Interdental & neweh fuerza \\
\hline 18 & $\tilde{\mathrm{N}}$ & $/ \mathrm{j} /$ & Como $\tilde{n}$ española & ñarki gato \\
\hline 19 & G & $/ \mathrm{y} /$ & $\begin{array}{l}\text { Equivalente a la secuencia } n g \text { del español, } \\
\text { pero como un solo sonido }\end{array}$ & $\begin{array}{l}\text { newehgen ser } \\
\text { fuerte }\end{array}$ \\
\hline 20 & $\mathrm{P}$ & $/ \mathrm{p} /$ & Como $p$ española & payun barba \\
\hline 21 & $\mathrm{R}$ & | & $\begin{array}{l}\text { Retrofleja. Se ensordece en territorio } \\
\text { huilliche }\end{array}$ & rvme junquillo \\
\hline 22 & $\mathrm{~S}$ & $/ \mathrm{s} /$ & Como $s$ del español de Chile & asukura azúcar \\
\hline 23 & $\mathrm{~T}$ & $/ t /$ & Como $t$ española & metawe cántaro \\
\hline 24 & - & $/ \mathrm{t} /$ & Interdental & - \\
\hline 25 & $\mathrm{X}$ & $/ \mathrm{ts} /$ & Un solo sonido, retroflejo & xawun reunión \\
\hline 26 & W & $/ \mathrm{w} /$ & $\begin{array}{l}\text { Como } g \text { en la palabra guante, pero más } \\
\text { suave }[\gamma] \\
\text { En diptongo, antes o después de otra vocal, } \\
\text { suena como } u\end{array}$ & $\begin{array}{l}\text { xawun reunión } \\
\text { awkiñko eco } \\
\text { pwel este (punto } \\
\text { cardinal) }\end{array}$ \\
\hline 27 & $\mathrm{Y}$ & $/ \mathrm{j} /{ }^{9}$ & $\begin{array}{l}\text { Antes de una vocal, como la } y \text { del español. } \\
\text { En diptongo suena como } i\end{array}$ & $\begin{array}{l}\text { yeku cormorán } \\
\text { koybatun mentir }\end{array}$ \\
\hline
\end{tabular}

Destacan las siguientes características de este alfabeto:

- Incorpora representaciones para la nasal dental y la lateral dental, pero no incluye una representación para el fonema oclusivo dental $/ \mathrm{t} /$.

- Intenta mantener un patrón de diferenciación entre mapuche y castellano, incorporando letras que no evocan la pronunciación del castellano cuando pudiera generarse transferencia lingüística. Por ejemplo, usa la letra $<\mathrm{x}>$ para representar el fonema africado retroflejo / $\mathrm{ts} /$ en vez de la opción $<$ tr $>$ propuesta en otros alfabetos.

- No sigue el patrón de diferenciación del castellano cuando propone la letra $<\mathrm{r}>$ para el fonema retroflejo $/ x /$.

- No incorpora representaciones que evidencien las variantes regionales periféricas, como la huilliche, aun cuando en sus datos biográficos se señala que recorrió diversas comunas haciendo trabajo de terreno lingüístico.

${ }^{9}$ Debido a que este fonema representa a tres sonidos [j], [j] y [j], hemos optado por el primero de ellos siguiendo a Sadowsky (Sadowsky, Scott et al) y a Salamanca (Salamanca, Gastón et al) 


\subsubsection{Alfabeto Mapuche Unificado}

En la presentación del Alfabeto Mapuche Unificado Mario Bernales (SOCHIL. Sociedad Chilena de Lingüística, 1988) señala — tal vez anticipando las discusiones futuras acerca de este tema— que:

La creación deliberada de un sistema de escritura para la lengua de una sociedad ágrafa tiene lugar dentro de un complejo de factores históricos y socioculturales y excede los problemas puramente técnicos de representación de una fonología por un alfabeto.

Mientras que los alfabetos de María Catrileo, de Adalberto Salas y del Instituto Lingüístico de Verano constituyeron la base para la formulación del Alfabeto Mapuche Unificado, el Alfabeto Raguileo quedó al margen, ya que su autor "mantuvo sin modificar su proposición original" (SOCHIL. Sociedad Chilena de Lingüística, 16). En el mismo texto, Gallardo (SOCHIL. Sociedad Chilena de Lingüística, 54-55) releva la baja estandarización del idioma mapuche y la propensión de algunos autores de alfabetos para la lengua mapuche a "una ortografia que quiere más mostrar cuán diferentes son las fonologías vernáculas de la fonología castellana que evocar de modo llano una estructura fonológica lo menos comprometida posible con un dialecto determinado".

Por su parte, Salas (SOCHIL. Sociedad Chilena de Lingüística, 66) reconoce como base del Alfabeto Mapuche Unificado el sistema fonológico del dialecto hablado en el valle central de la provincia de Cautín, la razón para ello está en la baja diferenciación fonológica entre los tres dialectos principales tradicionalmente reconocidos: el picunche o dialecto nortino; el pehuenche, de la precordillera de los Andes y mapuche central, conocido también como moluche. Salas señala además que la base fonológica que sustenta el Alfabeto Mapuche Unificado "No es aplicable al huilliche o dialecto sureño (de la provincia de Osorno)".

El Alfabeto Mapuche Unificado fue pensado desde una escolarización que alfabetiza en lengua castellana, y en un momento histórico en que los movimientos mapuches no manifestaban la fuerza reivindicatoria política actual. La propuesta alcanzó un alto nivel de difusión. Como señala Wittig (Wittig, 2006), "El hecho de que este alfabeto cuente con el respaldo de la comunidad académica propició su uso en la enseñanza del mapudungun".

El Alfabeto Mapuche Unificado (CONADI, 107) cuenta con 6 vocales y 21 consonantes (Tabla 2):

Tabla 2. Alfabeto Mapuche Unificado

\begin{tabular}{|l|l|l|l|l|}
\hline $\mathrm{N}^{\mathrm{o}}$ & Letras & Fonema & Pronunciación & Ejemplos \\
\hline 1 & $\mathrm{~A}$ & $/ \mathrm{a} /$ & Como vocal $a$ española. & awar habas \\
\hline 2 & $\mathrm{E}$ & $/ \mathrm{e} /$ & Como vocal $e$ española. & ñuke madre \\
\hline 3 & $\mathrm{I}$ & $/ \mathrm{i} /$ & Como vocal $i$ española. & koni entró \\
\hline 4 & $\mathrm{O}$ & $/ \mathrm{o} /$ & Como vocal $o$ española. & nor derecho \\
\hline 5 & $\mathrm{U}$ & $/ \mathrm{u} /$ & Como vocal $u$ española & uman dormir \\
\hline
\end{tabular}




\begin{tabular}{|c|c|c|c|c|}
\hline 6 & $\ddot{U}$ & $/ \mathbf{i} /$ & $\begin{array}{l}\text { Como } u \text {, sin redondear los labios } \\
\text { (variante [ə]) }\end{array}$ & kütral fuego \\
\hline 7 & $\mathrm{Ch}$ & $/ \mathrm{t} \int /$ & $\begin{array}{l}\text { Semejante al castellano ch. En el área } \\
\text { huilliche (sur) tiene también sonido } t s \text {. }\end{array}$ & challa olla \\
\hline 8 & $\mathrm{D}$ & $/ \theta /$ & $\begin{array}{l}\text { Al centro del territorio mapuche, como } \\
z \text { española. Al norte y sur, como } d \\
\text { suave [ð]. }\end{array}$ & deya hermana \\
\hline 9 & $\mathrm{~F}$ & $/ \mathrm{f} /$ & $\begin{array}{l}\text { Como } f \text { castellana (centro). } \\
\text { Más cercana a } v \text { (norte). } \\
\text { Como } f \text {, como } j \text { o aspirada (sur). }\end{array}$ & foro diente \\
\hline 10 & G & $/ \gamma /$ & Suena como $g$ suave $[\gamma]$. & legi apuntó \\
\hline 11 & $\mathrm{~K}$ & $/ \mathrm{k} /$ & $\begin{array}{l}\text { Como } c \text { española antes de vocales } a \text { o } \\
u \text {. }\end{array}$ & kachilla trigo \\
\hline 12 & $\mathrm{~L}$ & $/ 1 /$ & Como $l$ española. & lipang brazo \\
\hline 13 & $\underline{\mathrm{L}}$ & I/ & $\begin{array}{l}\text { Interdental. En territorio huilliche, } \\
\text { como retrofleja. }\end{array}$ & lawen remedio \\
\hline 14 & $\mathrm{Ll}$ & $|\Lambda|$ & $\begin{array}{l}\text { Como } l l \text { española más tradicional o } \\
\text { como habitualmente la } l l \text { de Chile. }\end{array}$ & llüngki rana \\
\hline 15 & $\mathrm{M}$ & $/ \mathrm{m} /$ & Como $m$ española. & mamüll madera \\
\hline 16 & $\mathrm{~N}$ & $/ \mathrm{n} /$ & Como $n$ española. & wentru hombre \\
\hline 17 & $\underline{\mathrm{N}}$ & $/ \mathrm{n} /$ & Interdental. & $\begin{array}{l}\text { kona empleado, } \\
\text { niño }\end{array}$ \\
\hline 18 & $\tilde{\mathrm{N}}$ & $/ \mathrm{n} /$ & Como $\tilde{n}$ española. & ñochi despacio \\
\hline 19 & $\mathrm{Ng}$ & $/ \mathrm{y} /$ & $\begin{array}{l}\text { Equivalente a la secuencia } n g \text { del } \\
\text { español, pero como un solo sonido. }\end{array}$ & ngan siembra \\
\hline 20 & $\mathrm{P}$ & $/ \mathrm{p} /$ & Como $p$ española & petu todavia \\
\hline 21 & $\mathrm{R}$ & $\mid$ & $\begin{array}{l}\text { Retrofleja. Se ensordece en territorio } \\
\text { huilliche. }\end{array}$ & rali plato \\
\hline 22 & $\mathrm{~S}$ & $/ \mathrm{s} /$ & Como $s$ del español de Chile. & sañwe cerdo \\
\hline 23 & $\mathrm{~T}$ & $/ \mathrm{t} /$ & Como $t$ española. & tüfa estela \\
\hline 24 & $\underline{T}$ & $/ \mathrm{t} /$ & Interdental & tol frente \\
\hline 25 & $\operatorname{Tr}$ & $/ \mathrm{ts} /$ & Un solo sonido, retroflejo. & fotra barro \\
\hline 26 & $\mathrm{~W}$ & $/ \mathrm{w} /$ & 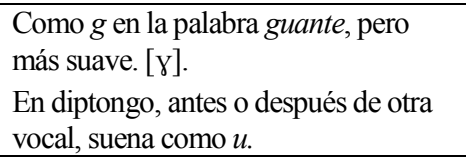 & $\begin{array}{l}\text { challwa pescado } \\
\text { pwel este (punto } \\
\text { cardinal) } \\
\text { chew dónde }\end{array}$ \\
\hline 27 & $\mathrm{Y}$ & $/ \mathrm{j} /$ & $\begin{array}{l}\text { Antes de una vocal, como la } y \text { del } \\
\text { español. } \\
\text { En diptongo suena como } i \text {. }\end{array}$ & $\begin{array}{l}\text { ayün gustar } \\
\text { doy más }\end{array}$ \\
\hline
\end{tabular}

Entre las características que pueden relevarse de este alfabeto se encuentran:

- Incorpora representaciones para las tres consonantes dentales.

- Se elabora a partir de la relación grafema-fonema ya establecida en el castellano, incorporando grafemas que evocan la pronunciación de la lengua castellana.

- No incorpora representaciones que evidencien las variantes regionales periféricas (por ejemplo, del pehuenche o del huilliche). 


\subsubsection{Grafemario Azümchefe}

Esta propuesta (CONADI, Azúmchefe. Estudio para la definición, 107) nace como intento de generar y legitimar un solo grafemario para el pueblo mapuche "que responda a la aspiración del sueño de las personas, de manera de transcribir la fonética de la lengua materna y que sirva como un medio de avance a la utopía del ser mapuche" (CONADI, Azümchefi. Grafemario único, 23). Basado en el análisis de seis grafemarios previos (Grafemario Antinao, Grafemario Cañulef, Grafemario Painequeo, Grafemario Huilcamán, Grafemario Raguileo y Grafemario Unificado), consta de 28 códigos lingüísticos o letras (6 vocales y 22 consonantes). Dos de las consonantes representan sonidos expresivos: uno afectivo y otro despectivo (Tabla 3):

Tabla 3. Grafemario Azümchefe

\begin{tabular}{|c|c|c|c|c|}
\hline $\mathrm{N}^{\mathrm{o}}$ & Letras & Fonema & Pronunciación & Ejemplos \\
\hline 1 & A & $/ \mathrm{a} /$ & Como vocal $a$ española. & az rostro \\
\hline 2 & $\mathrm{E}$ & /e/ & Como vocal $e$ española. & relmu arco iris \\
\hline 3 & $\mathrm{I}$ & $/ \mathrm{i} /$ & Como vocal $i$ española. & yien llevo \\
\hline 4 & $\mathrm{O}$ & $/ \mathrm{o} /$ & Como vocal $o$ española. & ofül sorbo \\
\hline 5 & $\mathrm{U}$ & $/ \mathrm{u} /$ & Como vocal $u$ española. & upen olvidar \\
\hline 6 & $\ddot{U}$ & $/ \mathbf{i} /$ & $\begin{array}{l}\text { Como } u \text { sin redondear los labios } \\
\text { (variante [ə]). }\end{array}$ & ürkün cansarse \\
\hline 7 & $\mathrm{Ch}$ & $/ \mathrm{t} f /$ & $\begin{array}{l}\text { Semejante al castellano } c h \text {. En el área } \\
\text { huilliche se utiliza también como } t s \text {. }\end{array}$ & chofü flojo \\
\hline 8 & $\mathrm{Z}$ & $/ \theta /$ & $\begin{array}{l}\text { Al centro, como zeta española. Al } \\
\text { norte y sur, como } d \text { suave [ð]. }\end{array}$ & zügu asunto \\
\hline 9 & $\mathrm{~F}$ & $/ \mathrm{f} /$ & $\begin{array}{l}\text { Como } f \text { castellana (centro). Más } \\
\text { cercana a } v \text { (norte). Como } f \text {, como } j \text { o } \\
\text { aspirada (sur). }\end{array}$ & faw aquí \\
\hline 10 & $\mathrm{Q}$ & $/ \gamma /$ & Como $g$ suave $[\gamma]$. & reqle siete \\
\hline 11 & K & $/ \mathrm{k} /$ & Como $c$ española antes de vocal $a$ o $u$. & Kuñiwün tener cuidado \\
\hline 12 & $\mathrm{~L}$ & $/ 1 /$ & Como $l$ española. & lolon hoyo \\
\hline 13 & Lh & 垈/ & $\begin{array}{l}\text { Interdental. En territorio huilliche, } \\
\text { como retrofleja. }\end{array}$ & kelhü rojo \\
\hline 14 & $\mathrm{Ll}$ & $\mid \mathrm{K} /$ & $\begin{array}{l}\text { Como } 11 \text { española más tradicional o } \\
\text { como habitualmente la } l l \text { de Chile. }\end{array}$ & llaskün enojarse \\
\hline 15 & $\mathrm{M}$ & $/ \mathrm{m} /$ & Como $m$ española. & mañüm agradecido \\
\hline 16 & $\mathrm{~N}$ & $/ \mathrm{n} /$ & Como $n$ española. & nütxam conversación \\
\hline 17 & $\mathrm{Nh}$ & $/ \mathrm{n} /$ & Interdental. & punh noche \\
\hline 18 & $\tilde{\mathrm{N}}$ & $/ \mathrm{n} /$ & Como $\tilde{n}$ española. & ñawe hija \\
\hline 19 & $\mathrm{G}$ & $/ \mathrm{y} /$ & $\begin{array}{l}\text { Equivalente a la secuencia } n g \text {, pero } \\
\text { como un solo sonido. }\end{array}$ & zugun hablar \\
\hline 20 & $\mathrm{P}$ & $/ \mathrm{p} /$ & Como $p$ española. & püran subir \\
\hline 21 & $\mathrm{R}$ & $\mid$ & $\begin{array}{l}\text { Retrofleja. Se ensordece en territorio } \\
\text { huilliche. }\end{array}$ & rapin vomitar \\
\hline 22 & $\mathrm{~S}$ & $/ \mathrm{s} /$ & Como $s$ del español de Chile. & weswes loquito \\
\hline 23 & $\mathrm{~T}$ & $/ \mathrm{t} /$ & Como $t$ española. & tapül hoja vegetal \\
\hline 24 & - & $/ \mathrm{t} /$ & Interdental. & - \\
\hline
\end{tabular}




\begin{tabular}{|c|c|c|c|c|}
\hline 25 & $\mathrm{Tx}$ & $/ \mathrm{ts} /$ & Un solo sonido, retroflejo. & txalka trueno \\
\hline 26 & $\mathrm{~W}$ & $/ \mathrm{w} /$ & $\begin{array}{l}\text { Como g en la palabra guante, pero más } \\
\text { suave. [४]. } \\
\text { En diptongo, después de otra vocal, } \\
\text { suena como } u \text {. }\end{array}$ & $\begin{array}{l}\text { weluñma al revés } \\
\text { füw hilo }\end{array}$ \\
\hline 27 & $\mathrm{Y}$ & $/ \mathrm{j} /$ & $\begin{array}{l}\text { Antes de vocal, como } y \text { del español. En } \\
\text { diptongo decreciente suena como } i \text {. }\end{array}$ & $\begin{array}{l}\text { yafkan ofender } \\
\text { üy nombre }\end{array}$ \\
\hline 28 & $\mathrm{SH}$ & [〕] & $\begin{array}{l}\text { Como la pronunciación fricativa, } \\
\text { bastante común (y estigmatizada) de ch } \\
\text { en castellano de Chile. }\end{array}$ & shomo mujercita querida \\
\hline 29 & $\mathrm{~T}^{\prime}$ & {$[\mathrm{t}]$} & $\begin{array}{l}\text { Más atrás que la } t \text { del castellano } \\
\text { (palatalizada). }\end{array}$ & $\begin{array}{l}\text { wesa t'e hombre malo (con } \\
\text { enfado) }\end{array}$ \\
\hline
\end{tabular}

Destacan las siguientes características de este alfabeto:

- Reconoce dos dentales que son graficadas con una H posterior (LH- NH). No reconoce la $\mathrm{T}$ dental.

- Esta propuesta incorpora representaciones gráficas para dos sonidos susceptibles de ser tratados como alófonos o como fonemas toda vez que su aparición implica una modificación de significado cuya magnitud, sin embargo, a nuestro juicio no satisface los criterios tradicionales para considerarlos fonemas distintos (al contrario de lo que plantea, con ciertas restricciones ${ }^{10}$, Sadowsky) en términos de significado formal, aunque sí en términos de significado pragmático, aunque []] incorpora una carga emotiva positiva y [ $\left.\mathrm{t}^{\mathrm{j}}\right]$ una carga negativa al enunciado.

- El fonema /ts/ es graficado como TX marcando con ello tanto la complejidad de la articulación del sonido como su singularidad frente a la secuencia tr del castellano.

- No incorpora representaciones que evidencien las variantes regionales periféricas (por ejemplo, del pehuenche o del huilliche).

2.1.4. Grafemario Universidad Católica de Temuco (Desiderio Catriquir y Gabriel Llanquinao)

El grafemario de Catriquir y Llanquinao de la UCT tiene el mérito de ser el resultado de hablantes nativos de la lengua mapuche con una praxis pedagógica reflexiva continua. Según comunicación personal de Catriquir en Solís (Solís), el diseño operó:

Sobre la base de los dos grafemarios revisados (Unificado, Raguileo), de mayor connotación del momento, como resultado de los debates para la uniformidad de los alfabetos para el mapuzugun, realizados en 1984, y en relación con las

\footnotetext{
10 "It should be noted that in some cases, the differences in the status of $/ \int /$ and $\left[\int\right]$ may be due to authors' differing interpretations of what constitutes a phoneme, rather than to actual dialectal differences" (Sadowsky, Scott et al, 92).
} 
corrientes de pensamiento que sustentan cada uno de ellos, en 1995 la Carrera de Pedagogía en Educación Básica Intercultural de la UCT se propone formular un grafemario para la enseñanza del mapuzugun en el contexto de la formación de profesores en educación intercultural. La formulación de grafemario wirilzugunwe contó con la participación de estudiantes bilingües mapuzuguncastellano de la carrera de Pedagogía en Educación Intercultural, profesores bilingües de la misma carrera.

Tabla 4. Grafemario UCT

\begin{tabular}{|c|c|c|c|c|}
\hline $\mathrm{N}^{\mathrm{o}}$ & Letras & Fonema & Pronunciación & Ejemplos $^{11}$ \\
\hline 1 & $\mathrm{~A}$ & $/ \mathrm{a} /$ & Como vocal $a$ española. & küpan venir \\
\hline 2 & $\mathrm{E}$ & $/ \mathrm{e} /$ & Como vocal $e$ española. & kexan sembrar \\
\hline 3 & I & /i/ & Como vocal $i$ española. & meli cuatro \\
\hline 4 & $\mathrm{O}$ & $/ \mathrm{o} /$ & Como vocal $o$ española. & zomo mujer \\
\hline 5 & $\mathrm{U}$ & $/ \mathrm{u} /$ & Como vocal $u$ española & ramtun preguntar \\
\hline 6 & $\ddot{U}$ & $/ \mathbf{i} /$ & $\begin{array}{l}\text { Como } u \text { sin redondear los labios } \\
\text { (variante [ə]) }\end{array}$ & küzaw trabajo \\
\hline 7 & $\mathrm{CH}$ & $/ \mathrm{t} \mathrm{d} /$ & $\begin{array}{l}\text { Semejante al castellano } c h \text {. En el área } \\
\text { huilliche se utiliza también como } t s \text {. }\end{array}$ & rukache dueño de casa \\
\hline 8 & $\mathrm{Z}$ & $/ \theta /$ & $\begin{array}{l}\text { Al centro, como z española. Al norte y } \\
\text { sur, como } d \text { suave [ð]. }\end{array}$ & mawiza montaña \\
\hline 9 & $\mathrm{~F}$ & $/ \mathrm{f} /$ & $\begin{array}{l}\text { Como } f \text { castellana (centro). } \\
\text { Más cercana a } v \text { (norte). } \\
\text { Como } f \text {, como } j \text { o aspirada (sur). }\end{array}$ & tüfa este \\
\hline 10 & Q & $/ \gamma /$ & Suena como $g$ suave $[\gamma]$. & naq abajo \\
\hline 11 & $\mathrm{~K}$ & $/ \mathrm{k} /$ & Como $c$ española antes de vocal $a$ o $u$. & kayu seis \\
\hline 12 & $\mathrm{~L}$ & $/ 1 /$ & Como $l$ española. & mülen haber \\
\hline 13 & $\mathrm{~B}$ & / $/$ & $\begin{array}{l}\text { Interdental. En territorio huilliche, } \\
\text { como retrofleja. }\end{array}$ & bewfü río \\
\hline 14 & $\mathrm{~J}$ & $\mid K /$ & $\begin{array}{l}\text { Como ll española más tradicional o } \\
\text { como habitualmente la } l l \text { de Chile. }\end{array}$ & mojfün sangre \\
\hline 15 & $\mathrm{M}$ & $/ \mathrm{m} /$ & Como $m$ española. & güxamkan conversar \\
\hline 16 & $\mathrm{~N}$ & $/ \mathrm{n} /$ & Como $n$ española. & wenxu hombre \\
\hline 17 & $\mathrm{H}$ & $/ \mathrm{n} /$ & Interdental. & kewüh lengua \\
\hline 18 & $\tilde{\mathrm{N}}$ & $/ \mathrm{n} /$ & Como $\tilde{n}$ española. & püñen hijos \\
\hline 19 & G & $/ \mathrm{y} /$ & $\begin{array}{l}\text { Equivalente a la secuencia } n g \text { del } \\
\text { español, pero como un solo sonido. }\end{array}$ & gürü zorro \\
\hline 20 & $\mathrm{P}$ & $/ \mathrm{p} /$ & Como $p$ española & epu $d o s$ \\
\hline 21 & $\mathrm{R}$ & $|x|$ & $\begin{array}{l}\text { Retrofleja. Se ensordece en territorio } \\
\text { huilliche. }\end{array}$ & kura piedra \\
\hline 22 & $\mathrm{~S}$ & $/ \mathrm{s} /$ & Como $s$ del español de Chile. & magsun buey \\
\hline 23 & $\mathrm{~T}$ & $/ \mathrm{t} /$ & Como $t$ española. & fotüm hijos \\
\hline 24 & - & $/ \mathrm{t} /$ & Interdental. & - \\
\hline 25 & $\mathrm{X}$ & $/ \mathrm{ts} /$ & Retroflejo. & xawün reunión \\
\hline
\end{tabular}

${ }^{11}$ La mayor parte de estos ejemplos están tomados de los textos para enseñanza de lengua mapuche de Catriquir et al. (Catriquir, Desiderio; Huenchulaf, Rosa; y Sandoval, Cristián). 


\begin{tabular}{|l|l|l|l|l|}
\hline 26 & $\mathrm{~W}$ & $/ \mathrm{w} /$ & $\begin{array}{l}\text { Como g en la palabra guante, pero más } \\
\text { suave. }\end{array}$ & wiji sur \\
\hline 27 & $\mathrm{Y}$ & $/ \mathrm{j} /$ & $\begin{array}{l}\text { Antes de una vocal, como la } y \text { del } \\
\text { español. Después de vocal, suena } \\
\text { como } i .\end{array}$ & zeya hermana \\
\hline 28 & $\mathrm{SH}$ & $/ \mathrm{J} /$ & $\begin{array}{l}\text { Como la pronunciación fricativa, } \\
\text { bastante común (y estigmatizada) de ch } \\
\text { en castellano de Chile. }\end{array}$ & ufisha oveja \\
\hline
\end{tabular}

Algunos comentarios que cabe hacer respecto de este alfabeto son los siguientes:

- Igual que el grafemario de Raguileo, incorpora representaciones para la nasal dental y la lateral dental, pero no incluye una representación para el fonema oclusivo dental $/ \mathrm{t} /$.

- Este alfabeto soluciona - mediante la propuesta de la letra $\mathrm{j}$ - el problema planteado por Salas (SOCHIL. Sociedad Chilena de Lingüística, 84):

El digrama $<11>$ es algo más problemático, ya que el fonema $/ /$ puede ocurrir en posición prevocálica o postvocálica, y si se lo representa por $<1>$, se puede tener un grupo ortográfico $>1 \mathrm{l}>$ que corresponde a $<\mathrm{l}>+<\mathrm{l}$, una de la coda de una sílaba y la otra del frontis de la sílaba siguiente, y no al fonema $/ \lambda$.

- Elaborado de acuerdo con la pronunciación de las variantes geográficas o dialectales más prestigiosas del mapuche, no da cuenta de las posibilidades que reclaman los hablantes de dialectos más sureños.

\subsubsection{Grafemario Cacicado Huilliche}

Resultado de una preocupación del cacicado ${ }^{12}$ (Junta General de Caciques, 2000) por aumentar el número de hablantes huilliches, este grafemario retoma el trabajo iniciado por el grupo Monku Küsobkien en la década de los 80 .

Tabla 5. Grafemario Cacicado Huilliche

\begin{tabular}{|l|l|l|l|l|}
\hline $\mathrm{N}^{\mathrm{o}}$ & Letras & Fonema & Pronunciación & Ejemplos \\
\hline 1 & $\mathrm{~A}$ & $/ \mathrm{a} /$ & Como vocal $a$ española. & axun cansado \\
\hline 2 & $\mathrm{E}$ & $/ \mathrm{e} /$ & Como vocal $e$ española. & eitimo alli \\
\hline 3 & $\mathrm{I}$ & $/ \mathrm{i} /$ & Como vocal $i$ española. & ince yo \\
\hline 4 & $\mathrm{O}$ & $/ \mathrm{o} /$ & Como vocal $o$ española. & ofuld chupar \\
\hline 5 & $\mathrm{U}$ & $/ \mathrm{u} /$ & Como vocal $u$ española & uñun bonito \\
\hline 6 & $\mathrm{~V}$ & $/ \mathrm{i} /$ & $\begin{array}{l}\text { Como } u \text { sin redondear los labios } \\
\text { (variante }[\partial] \text { ) }\end{array}$ & antv día, sol \\
\hline 7 & $\mathrm{C}$ & $/ \mathrm{t} / /$ & $\begin{array}{l}\text { Semejante al castellano } \text { ch. En el } \\
\text { área huilliche se utiliza también } \\
\text { como } t s .\end{array}$ & cag junto \\
\hline
\end{tabular}

${ }^{12}$ El cacicado huilliche comprende la organización política de la Fütawillimapu ('grandes tierras del sur'), mediante la Junta General de Caciques de la Fútawillimapu dirigida por el Lonko Mayor Antonio Alcafuz Canquil. 


\begin{tabular}{|c|c|c|c|c|}
\hline 8 & $\begin{array}{l}\mathrm{Z} \\
\mathrm{D}\end{array}$ & $/ \theta /$ & $\begin{array}{l}\text { Al centro, como } z \text { española. Al norte } \\
\text { y sur, como } d \text { suave [ð]. }\end{array}$ & $\begin{array}{l}\text { ziven compañero } \\
\text { duam intención }\end{array}$ \\
\hline 9 & $\begin{array}{l}\text { F } \\
\text { J } \\
B \\
H\end{array}$ & $/ \mathrm{f} /$ & $\begin{array}{l}\text { Como } f \text { castellana (centro). Más } \\
\text { cercana a } v \text { (norte). Como } f \text {, como } j \text { o } \\
\text { aspirada (sur). }\end{array}$ & $\begin{array}{l}\text { fane pesado } \\
\text { joxum hijo } \\
\text { haku pellejo }\end{array}$ \\
\hline 10 & $\mathrm{Q}$ & $/ \gamma /$ & Como $g$ suave $[\gamma]$. & liq blanco \\
\hline 11 & $\mathrm{~K}$ & $/ \mathrm{k} /$ & Como $c$ española antes de vocal $a \mathrm{o} u$. & kalfu azul \\
\hline 12 & $\mathrm{~L}$ & $/ 1 /$ & Como $l$ española. & lapa paloma \\
\hline 13 & - & ג/ & $\begin{array}{l}\text { Interdental. En huilliche, como } \\
\text { retrofleja. }\end{array}$ & - \\
\hline 14 & $\mathrm{Y}$ & $/ / \mathrm{K} /$ & $\begin{array}{l}\text { Como ll española más tradicional o } \\
\text { como habitualmente la } l l \text { de Chile. }\end{array}$ & yum oculto \\
\hline 15 & $\mathrm{M}$ & $/ \mathrm{m} /$ & Como $m$ española. & miaun andar \\
\hline 16 & $\mathrm{~N}$ & $/ \mathrm{n} /$ & Como $n$ española. & namun pie \\
\hline 17 & - & $/ \mathrm{n} /$ & Interdental. & - \\
\hline 18 & $\tilde{\mathrm{N}}$ & $/ \mathrm{n} /$ & Como $\tilde{n}$ española. & ñapud nieve \\
\hline 19 & $\mathrm{G}$ & $/ \mathrm{y} /$ & $\begin{array}{l}\text { Equivalente a la secuencia } n g \text {, pero } \\
\text { como un solo sonido. }\end{array}$ & gal ojalá \\
\hline 20 & $\mathrm{P}$ & $/ \mathrm{p} /$ & Como $p$ española & pafuld salir sangre \\
\hline 21 & $\mathrm{R}$ & / & $\begin{array}{l}\text { Retrofleja. Se ensordece en territorio } \\
\text { huilliche. }\end{array}$ & ruka casa \\
\hline 22 & $\mathrm{~S}$ & $/ \mathrm{s} /$ & Como $s$ del español de Chile. & sumun hablar \\
\hline 23 & $\mathrm{~T}$ & $/ \mathrm{t} /$ & Como $t$ española. & tapel hoja \\
\hline 24 & - & $/ \mathrm{t} /$ & Interdental & - \\
\hline 25 & $\mathrm{X}$ & $/ \mathrm{ts} /$ & Retroflejo. & axun cansado \\
\hline 26 & $\mathrm{~W}$ & $/ \mathrm{w} /$ & "Como la w del inglés" & $?$ \\
\hline 27 & $\mathrm{Y}$ & $/ \mathrm{j} /$ & $\begin{array}{l}\text { Antes de vocal, como } y \text { del español. } \\
\text { Después de vocal como } i\end{array}$ & yum oculto \\
\hline
\end{tabular}

Comentarios a este alfabeto:

- No proporciona representación para las consonantes dentales $/ \mathrm{d} /, \mathrm{n} / \mathrm{ni} / \mathrm{t} / \mathrm{Se}$ desliza, sin embargo, el uso de LD en "pafuld" (salir sangre) para la lateral interdental.

- A diferencia de los anteriores grafemarios donde las correspondencias fonema/grafema tienden a la univocidad, se observa aquí una coexistencia de soluciones que implican también posibilidades de múltiples representaciones grafémicas para un mismo fonema, como se manifiesta en las cuatro realizaciones grafémicas propuestas para el fonema/f/ y en las dos para el fonema $/ \theta /$, hecho que evidencia un esfuerzo por representar las variaciones alofónicas que caracterizan el habla huilliche.

\footnotetext{
${ }^{13}$ Explicación tomada del mismo texto del cacicado.
} 
- No incluye representación grafémica especial para $/ K /$, lo que da cuenta de la asimilación de este fonema a su realización en el castellano de Chile por efecto del yeísmo.

\subsubsection{Grafemario Salvador Rumian}

Salvador Rumian es hijo de Ponciano Rumian, reconocido líder e investigador huilliche, activo participante del grupo Monku Küsobkien en la década de los 80 que mostró especial interés por la revitalización de la lengua en el territorio sureño. Heredero de este ambiente, Salvador ha realizado cursos de lengua y de historia mapuche cuyos materiales están disponibles en Internet. Su propuesta de grafemario (Rumian, 2011) reconoce, desde el nombre de la variante regional Che Süngun, la fonología dialectal sureña que sustenta la propuesta.

Tabla 6. Grafemario para el Che Süngun (Salvador Rumian)

\begin{tabular}{|c|c|c|c|c|}
\hline $\mathrm{N}^{\mathrm{o}}$ & Letras & Fonema & Pronunciación & Ejemplos del autor \\
\hline 1 & $\mathrm{~A}$ & $/ \mathrm{a} /$ & Como vocal $a$ española. & alwe \\
\hline 2 & $\mathrm{E}$ & /e/ & Como vocal $e$ española. & etsiun \\
\hline 3 & $\mathrm{I}$ & $/ \mathrm{i} /$ & Como vocal $i$ española. & imiln \\
\hline 4 & $\mathrm{O}$ & $/ \mathrm{o} /$ & Como vocal $o$ española. & opun \\
\hline 5 & $\mathrm{U}$ & $/ \mathrm{u} /$ & Como vocal $u$ española & uñuñ \\
\hline 6 & $\ddot{\mathrm{U}}$ & $/ \mathbf{i} /$ & $\begin{array}{l}\text { Como } u \text { sin redondear los labios } \\
\text { (variante [ə]) }\end{array}$ & ül'kantun \\
\hline 7 & $\begin{array}{l}\text { Ch } \\
\text { Ts }\end{array}$ & $/ \mathrm{t} \int /$ & $\begin{array}{l}\text { Semejante al castellano } c h \text {. En el } \\
\text { área williche se utiliza también } \\
\text { como } t s \text {. }\end{array}$ & $\begin{array}{l}\text { chaiwe } \\
\text { tsasro }\end{array}$ \\
\hline 8 & $\begin{array}{l}\text { D (no oficial) } \\
\text { S } \\
\text { SH }\end{array}$ & $/ \theta /$ & $\begin{array}{l}\text { Al centro, como zeta española. Al } \\
\text { norte y sur, como } d \text { suave [ð]. }\end{array}$ & $\begin{array}{l}\text { mawidam } \\
\text { süngun } \\
\text { kishu }\end{array}$ \\
\hline 9 & $\begin{array}{l}\mathrm{F} \\
\mathrm{V} \text { (no oficial) } \\
\mathrm{J}\end{array}$ & $/ \mathbf{f} /$ & $\begin{array}{l}\text { Como } f \text { castellana (centro). Más } \\
\text { cercana a } v \text { (norte). Como } f \text {, como } j \\
\text { o aspirada (sur). }\end{array}$ & $\begin{array}{l}\text { folil } \\
\text { kuyiva } \\
\text { josrü }\end{array}$ \\
\hline 10 & G & $/ \gamma /$ & Como $g$ suave $[\gamma]$. & srag \\
\hline 11 & $\mathrm{~K}$ & $/ \mathrm{k} /$ & Como $c$ española antes de vocal $a \mathrm{o} u$. & kasra \\
\hline 12 & $\mathrm{~L}$ & $/ 1 /$ & Como $l$ española. & lüf \\
\hline 13 & $\mathrm{~L}^{\prime}$ & N/ & $\begin{array}{l}\text { Interdental. } \\
\text { En territorio huilliche, como } \\
\text { retrofleja, }\end{array}$ & l'afken \\
\hline 14 & $\mathrm{Ll}$ & $\mid \overline{\mid K}$ & $\begin{array}{l}\text { Como ll española más tradicional o } \\
\text { como habitualmente la } l l \text { de Chile. }\end{array}$ & llüfken \\
\hline 15 & $\mathrm{M}$ & $/ \mathrm{m} /$ & Como $m$ española. & mapu \\
\hline 16 & $\mathrm{~N}$ & $/ \mathrm{n} /$ & Como $n$ española. & nag \\
\hline 17 & $\mathrm{~N}^{\prime}$ & $/ \mathrm{n} /$ & Interdental. & n'amun' \\
\hline 18 & $\tilde{\mathrm{N}}$ & $/ \mathrm{n} /$ & Como $\tilde{n}$ española. & ñasrki \\
\hline 19 & $\mathrm{Ng}$ & $/ \mathrm{y} /$ & $\begin{array}{l}\text { Equivalente a la secuencia } n g \text {, pero } \\
\text { como un solo sonido. }\end{array}$ & ngisre \\
\hline
\end{tabular}




\begin{tabular}{|l|l|l|l|l|}
\hline 20 & $\mathrm{P}$ & $/ \mathrm{p} /$ & Como $p$ española & pül'ko \\
\hline 21 & $\mathrm{Sr}(\mathrm{r})$ & $/ \mathrm{s} /$ & $\begin{array}{l}\text { Retrofleja. Se ensordece en } \\
\text { territorio huilliche. }\end{array}$ & srakisuam \\
\hline 22 & $\mathrm{~S}$ & $/ \mathrm{s} /$ & Como $s$ del español de Chile. & süngun \\
\hline 23 & $\mathrm{~T}$ & $/ \mathrm{t} /$ & Como $t$ española. & tunten \\
\hline 24 & $\mathrm{~T}$ & $/ \mathrm{t} /$ & Interdental. & t'eümel' \\
\hline 25 & $\mathrm{Tr}$ & $/ \mathrm{ts} /$ & Retroflejo. & trufken \\
\hline 26 & $\mathrm{~W}$ & $/ \mathrm{w} /$ & $\begin{array}{l}\text { Como g en la palabra guante, pero } \\
\text { más suave. }\end{array}$ & waiwen \\
\hline 27 & $\mathrm{Y}$ & $/ \mathrm{j} /$ & $\begin{array}{l}\text { Antes de vocal, como } y \text { del } \\
\text { español. Después de vocal como } i .\end{array}$ & yom \\
\hline
\end{tabular}

Los aspectos que destacan en esta propuesta son:

- Incluye representaciones específicas para las consonantes dentales $/ 1 /, / n /$ y $/ t /$, lo que marca mediante apóstrofe.

- Como en el anterior grafemario (cacicado), se observa aquí la presencia de soluciones no unívocas para representar un mismo fonema: $\mathrm{D}, \mathrm{S}$ y SH para $/ \theta /, \mathrm{Ch}$ y TS para /t $\mathrm{f} / \mathrm{y}$ F, V y J para/f/, lo que probablemente obedece al mismo esfuerzo por representar las variaciones alofónicas que caracterizan el habla huilliche.

- Incluye dos consonantes (D y V) bajo el rótulo de "grafemas no oficiales" para identificar fonemas que considera "usados de manera marginal por los hablantes, debido a una altamente probable influencia del español" (Rumian 2011). La letra $\mathrm{D}$ aparece representando el fonema $/ \theta /$ (dado el ejemplo mawidam) junto a las letras $\mathrm{S}$ y SH, lo que evidencia la sustitución del sonido original $[\theta]$ por [s] y [S] aunque generalmente en distintos contextos. La letra $\mathrm{V}$ representa, en realidad, una pronunciación de /f/ poco frecuente en la zona huilliche.

\section{COMENTARIOS FINALES}

Hemos trabajado con dos conjuntos de propuestas que no miran exactamente hacia el mismo objeto. Un primer grupo enfocado en el mapuche central y un segundo grupo que emprende la tarea de plantear un alfabeto más representativo de lo propio aunque sobre la base de lo ya construido previamente para una lengua mapuche que tiende a la estandarización dialectal. Esta panorámica nos permite las siguientes consideraciones:

Tensiones sociopoliticas. Hay propuestas que buscan alejarse de la fonética del castellano para mantener los rasgos más diferenciadores y propios del mapuzugun. Es la marca que deja el grafemario de Raguileo para otros posteriores. Se advierte en estas propuestas un movimiento contrario a la asimilación, una lealtad lingüística en la que subyace la teoría del conflicto lingüístico. Las lenguas no son ajenas a los intereses antagónicos de las comunidades y suelen confrontarse social y políticamente. La lengua dominada intenta ofrecer resistencia a los patrones de dominación. Se defiende la lengua como soporte y símbolo de la identidad y cultura colectiva, es decir, como 
recurso simbólico esencial para la identificación y representación del grupo o pueblo que la habla. Están en esta situación los grafemarios de Raguileo, Catriquir y, en menor grado, los del cacicado y Salvador Rumian; estos dos últimos más concentrados en visibilizar los rasgos característicos del habla regional huilliche por contraste con el centro lingüístico mapuche.

Tensiones territoriales. El énfasis en las propuestas más localistas está puesto en la defensa de las variedades dialectales frente a las variedades más vitales y prestigiosas de la lengua mapuche. Aunque subyace también una tensión política, esta es de carácter interno y se vincula con un rechazo a la hegemonía del centro mapuche, tanto en materias lingüísticas como culturales. En los grafemarios más locales se opera por sobrediferenciación, el resultado es que varios grafemas pueden representar un solo fonema, pues se destacan las representaciones alofónicas. En algunos casos, junto a las letras del código dominante, como TS junto a $\mathrm{CH}$ para /t $\mathrm{f} / ; \mathrm{V} / \mathrm{J} / \mathrm{H}$ junto a $\mathrm{F}$ para /f/; y, en otros casos, reemplazando la letra del código dominante como SR que sustituye a $R$.

Los grafemarios huilliches, cuando no se proponen realzar sus particulares modos de pronunciación, se nutren de diferentes propuestas previas, por ejemplo, a veces incorporan letras del Grafemario Raguileo: V para la vocal y X en el grafemario del cacicado y $\mathrm{G}$ en el del cacicado y en el de Rumian. En otros casos, siguen el Alfabeto Unificado, como en la elección de Tr y Ng del grafemario de Rumian.

Llama la atención que, en general, mientras los grafemarios basados en los dialectos central, pehuenche y nortino de la lengua mapuche coinciden en usar R para la representación de $/ \mathrm{x} /$, descuidando el criterio de diferenciación con el castellano que subyace en sus propuestas, el grafemario de Salvador Rumian utiliza SR para representar el mismo fonema, buscando reflejar con ello la pronunciación de esta variante sorda en el habla sureña.

Como se ha señalado, las elecciones gráficas para el área huilliche relevan la diferencia territorial más que la diferencia con la lengua dominante, lo que puede explicarse por el intenso proceso de reetnización de grupos cuya variedad dialectal se ha visto decididamente amenazada en las últimas décadas y por una actitud defensiva frente a la estigmatización que estas hablas han recibido desde el centro lingüístico mapuche.

Sobre algunas concreciones. En la práctica, el problema se presenta en los hablantes de mayor edad que escriben "como pronuncian" y que en áreas de menor vitalidad son responsables de enseñar y traspasar la lengua a las nuevas generaciones. Las vacilaciones en el uso de alfabetos afecta también a las educadoras mapuches que, por una parte, deben utilizar el Azümchefe de acuerdo con los planes y programas ministeriales y, por otra parte, estudian con docentes que enseñan con diferentes modos de escritura, a la vez que reciben presión de las comunidades locales para utilizar una representación no "exógena" de la lengua. 
Relación entre organización política y "administración” de la lengua. La organización política del pueblo mapuche ha sido tradicionalmente no jerarquizada. Incluso, en el caso del cacicado mapuche, el más parecido a la cultura occidental por su carácter jerárquico piramidal, el Lonko Mayor bajo el cual se encuentran varios cacicados de la Fütawillimapu, no tiene poder directo para intervenir en decisiones de los otros cacicados y su intervención se enmarca en acuerdos, generalmente tomados en los Txawün o reuniones de los Lonko.

Desde una mirada occidental, la decisión por un alfabeto único aparece como impostergable, sin embargo, es posible que desde la perspectiva mapuche no resulte tan importante contar con un solo alfabeto, sino que prime el respeto por las territorialidades, como ha ocurrido en su organización política, incluso frente al inminente peligro actual de dominación winka. Después de todo, Salas (SOCHIL, 1988), al referirse a la escritura de las consonantes epénticas, reconoció la posibilidad de postergar decisiones y dejar alternativas paralelas a un problema cuando no hay conflictos entre lo estimado técnicamente como más adecuado y lo que ocurre en la práctica:

En principio estos desarrollos consonánticos no deberían ser representados en una ortografía estrictamente fonémica. Sin embargo se ha observado que para algunos hablantes nativos el desarrollo consonántico forma parte de la palabra, como un segmento constituyente más y la sienten mutilada al escucharla o verla escrita con la sola vocal inicial. Se ha observado que tienden a representarlo al escribir y se desconciertan si se les pide que no lo hagan. En consecuencia, es aconsejable, al menos en las etapas iniciales del proceso de alfabetización, aceptarles la representación de la fricativa epéntica, aun cuando se introduzcan alternancias (...). Salas (SOCHIL, 101).

Universidad de Los Lagos* Departamento de Humanidades y Artes Av. Fuchslocher 1305, Osorno (Chile) palvarez@ulagos.cl

Universidad de Los Lagos**

Departamento de Educación Av. Fuchslocher 1305, Osorno (Chile) aforno@ulagos.cl

Universidad de Los Lagos*** Departamento de Humanidades y Artes Av. Fuchslocher 1305, Osorno (Chile) erisco@ulagos.cl 
Pilar Álvarez-Santullano, Amilcar Forno y Eduardo Risco

\section{OBRAS CITADAS}

Abraham, W. Diccionario de Terminología Lingüistica Actual. Madrid: Gredos, 1981. Catriquir, Desiderio; Huenchulaf, Rosa. y Sandoval, Cristián. Curso taller de Aprendizaje Mapunzungun. Tomos 1, 2 y 3. Termuco: Universidad Católica de Temuco, 2012.

CONADI. Azúmchefe. Grafemario único del idioma mapuche. Estudio para la definición de un grafemario para la lengua mapuche. Temuco: Departamento de Cultura y Educación, CONADI, 2008.

_ AZÜMCHEFI. Grafemario Único del idioma mapuche. Departamento de Cultura y Educación. Santiago de Chile: CONADI Nacional, Ministerio de Planificación y Cooperación, Gobierno de Chile, 1999.

De Valdivia, Luis. Arte y Gramática General de la Lengva qve corre en todo el reyno de Chile, con vn vocabulario y confessonario: Compuestos por el Padre Luis de Valdivia de la Compañía de Jesus, en la Provincia del Perú. Sevilla, 1684.

Junta General de Caciques. Documento de trabajo. Butalmapu. Osorno, Chile, 2000.

Kintupil, Erwin. "Saltapura" 2007. Anselmo Raguileo. 2 de febrero de 2015. Disponible en: http://trawunsaltapura.blogia.com/2007/071805-anselmo-raguileo.php

Rumian, Salvador. Futawillimapu.org. 2011. noviembre de 2012. Disponible en: http://www.futawillimapu.org/pub/2011/PESPI/PESPI_2011_-_Che_Sungun_S01.pdf

Sadowsky, Scott et al. "Illustrations of the IPA. Mapudungun", Journal of the International Phonetic Association (2013): 87-96.

Salamanca, Gastón et al. "Mapuche hablado en Melipeuco: Fonemas segmentales, fonotaxis y comparación con otras variedades", LOGOS Revista de Lingüística, Filosofia y Literatura (2009): 74-95.

SOCHIL. Sociedad Chilena de Lingüística. Alfabeto Mapuche Unificado. Temuco: Universidad Católica de Temuco, 1988.

Solís, Marcia. Análisis de los grafemarios propuestos para la escritura de la lengua mapuche. Tesis de grado para optar al título profesional de Profesora de Lengua Castellana y Comunicaciones. Universidad de Los Lagos, Osorno, 2013.

Wittig, Fernando. La escritura en mapudungun: alfabetos en uso y nuevos escenarios. CISAI. Centro interdipartimentale di studi sull'america indigena. Università Degli Studi di Siena" (2006). Rescatado el 2 de febrero de 2015. Disponible en: http://www.unisi.it/cisai/arealingtesti.htm

Zúñiga, Fernando. Mapudungun. El habla mapuche. Introducción a la lengua mapuche con notas comparativas y un $C D$. Santiago de Chile: Centro de Estudios Públicos, 2006. 